Document downloaded from:

http://hdl.handle.net/10251/99663

This paper must be cited as:

Cordero Barbero, A.; Franques, A.; Torregrosa Sánchez, JR. (2016). Chaos and convergence of a family generalizing Homeier's method with damping parameters. Nonlinear Dynamics. 85(3):1939-1954. doi:10.1007/s11071-016-2807-0

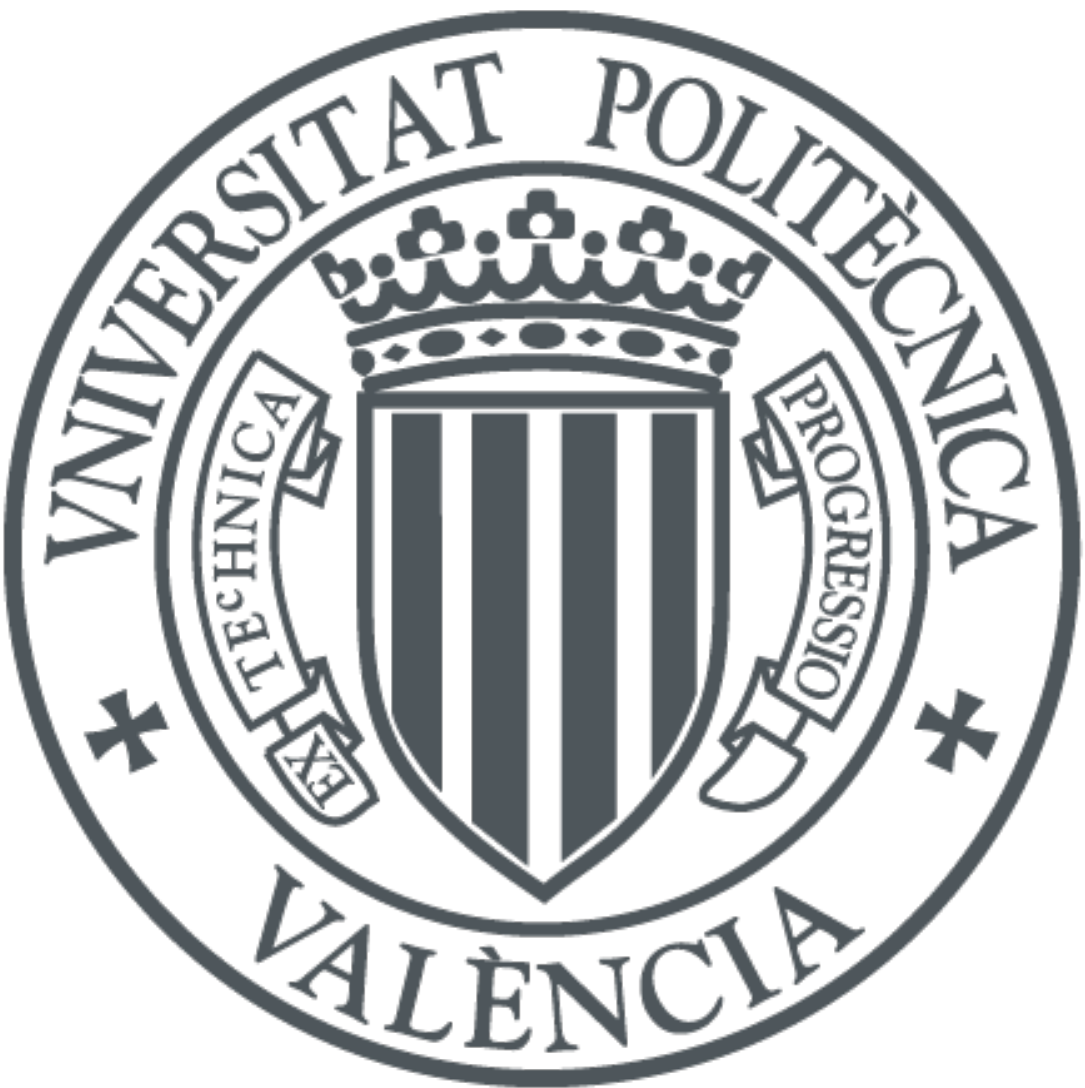

The final publication is available at

http://doi.org/10.1007/s11071-016-2807-0

Copyright Springer-Verlag

Additional Information 


\title{
Chaos and Convergence of a family generalizing Homeier's method with damping parameters *
}

\author{
$\underline{\text { Alicia Cordero }} \stackrel{\text { Antonio Franques, Juan R.Torregrosa }}{\text { Aut }}$ \\ Instituto Universitario de Matemática Multidisciplinar \\ Universitat Politècnica de València \\ Camino de Vera s/n, 46022 València, Spain
}

May 3, 2016

\begin{abstract}
In this paper, a family of parametric iterative methods for solving nonlinear equations, including Homeier's scheme, is presented. Its local convergence is obtained and the dynamical behavior on quadratic polynomials of the resulting family is studied in order to choose those values of the parameter that ensure stable behavior. To get this aim, the analysis of fixed and critical points and the associated parameter plane show the dynamical richness of the family and allow us to find members of this class with good numerical properties and also other ones with pathological conduct. To check the stable behavior of the good selected ones the discretized planar 1D-Bratu problem is solved. Some of those chosen members of the family achieve good results when Homeier scheme fails.

Nonlinear equations, iterative methods, dynamical behavior, parameter plane, convergence regions, Bratu problem
\end{abstract}

\section{Introduction}

The design and analysis of new iterative methods for obtaining the roots of a nonlinear equation $f(x)=0$, where $f: I \subset \mathbb{R} \rightarrow \mathbb{R}$ is a real function defined in an open interval $I$, is an active area of research in Numerical Analysis (see the survey [26] and the references therein). In the last decades, many iterative schemes of increasing order of convergence have been developed and have shown their efficiency, in numerical terms (see, for example $[5,11,28])$. However, lower order classical methods have been usually revisited only trying to increase its order of convergence. Recently, the idea of taking into account not only the order and efficiency of the methods, but also the stability of the resulting schemes, is gaining strength.

The starting point of this study is the third-order iterative scheme

$$
\begin{aligned}
y_{k} & =x_{k}-\frac{f\left(x_{k}\right)}{f^{\prime}\left(x_{k}\right)}, \\
x_{k+1} & =x_{k}-\frac{1}{2}\left[\frac{f\left(x_{k}\right)}{f^{\prime}\left(x_{k}\right)}+\frac{f\left(x_{k}\right)}{f^{\prime}\left(y_{k}\right)}\right],
\end{aligned}
$$

which was presented by Homeier in [18]. Let us remark that the first step of this scheme is the well-known second-order Newton's method, which is the most used procedure in practice.

In this paper, we design a family of parametric iterative methods including Homeier's method (1). An infinite number of elements of this class holds the convergence velocity of Homeier's scheme, preserving and even improving its stability properties. The iterative expression of this family is

$$
\begin{aligned}
& y_{k}=x_{k}-\varphi \frac{f\left(x_{k}\right)}{f^{\prime}\left(x_{k}\right)}, \\
& x_{k+1}=x_{k}-\left[\gamma \frac{f\left(x_{k}\right)}{f^{\prime}\left(y_{k}\right)}+\delta \frac{f\left(x_{k}\right)}{f^{\prime}\left(x_{k}\right)}\right],
\end{aligned}
$$

\footnotetext{
${ }^{*}$ This research was supported by Ministerio de Economía y Competitividad MTM2014-52016-C02-2-P.
}

†Email: acordero@mat.upv.es 
where $\varphi, \gamma$ and $\delta$ are free disposable parameters, that have been introduced in an intuitive way in order to improve the convergence and stability of Homeier's method. Let us notice that, if we choose $\gamma=\frac{1}{2}, \varphi=1$ and $\delta=\frac{1}{2}$ we get the method designed by Homeier.

The aim of this manuscript is to analyze, by using complex dynamical tools, the stability of the elements of this family on quadratic polynomials. This qualitative study will allow us to select those members without convergence anomalies. In fact, when an iterative method fails for low-degree polynomials, it usually has unstable behavior on many nonlinear equations. We also make a quantitative analysis by applying some elements of the family, including Homeier's scheme, for solving the nonlinear system obtained by discretizing the planar 1DBratu problem.

The rest of the paper is organized as follows: in Section 2 we study the local convergence of family (2) of iterative methods, proving the third-order of convergence under some conditions on the parameters. So, an infinite quantity of members of this class has third order of convergence. We also compare their efficiency with classical Newton's scheme and analyze the obtained error equation to minimize the asymptotic error. In order to select the most stable members of (2), in Section 3 we present the dynamical behavior of the rational operator associated to this family on quadratic polynomials. The fixed and critical points of this operator are analyzed as well as the stability of the fixed points. We also show the stability regions of the fixed points and the attractive 2-periodic orbits, whose analytical expression is found in terms of parameter $\gamma$. As a result, we get wider regions of stability than in other classical higher-order classes such as King's [12] or Jarratt-type families [22]. Section 4 is devoted to describe a quantitative study, showing the performance of some stable members of the class on planar 1D-Bratu problem. Finally, some conclusions and the references used in this manuscript are shown.

\section{Convergence analysis}

In this section we present the results related with the convergence and efficiency of family (2).

We recall that a sequence $\left\{x_{k}\right\}_{k \geq 0}$ converges to $\alpha$ with order of convergence $p$ if there exists a positive constant $C$ such that

$$
\lim _{k \rightarrow \infty} \frac{\left|x_{k+1}-\alpha\right|}{\left|x_{k}-\alpha\right|^{p}}=C
$$

and in this case the error equation of the method can be expressed as

$$
e_{k+1}=C e_{k}^{p}+O\left(e_{k}^{p+1}\right)
$$

where $e_{k}=x_{k}-\alpha$ and $C$ is the asymptotic constant error. In particular, if $p=1(C<1)$ the convergence is called linear, quadratic if $p=2$, cubic if $p=3$ and so on.

In the following result the sufficient conditions that assure the third-order convergence of family (2) are established.

Theorem 1 Let $\alpha \in I$ be a simple zero of a sufficiently differentiable function $f: I \subset \mathbb{R} \rightarrow \mathbb{R}$ in an open interval $I$ and $x_{0}$ an initial guess close enough to $\alpha$. Methods defined in (2) have third-order of convergence if $\varphi=\frac{1}{2 \gamma}$ and $\delta=1-\gamma$, where $\gamma \neq 0$. Their error equation is

$$
e_{k+1}=\left[\left(8 \gamma-3-4 \gamma^{2}\right) c_{2}^{2}+\left(\frac{11}{4}-6 \gamma+3 \gamma^{2}\right) c_{3}\right] e_{k}^{3}+O\left(e_{k}^{4}\right)
$$

where $c_{k}=\frac{1}{k !} \frac{f^{(k)}(\alpha)}{f^{\prime}(\alpha)}, k=2,3, \ldots$ and $e_{k}=x_{k}-\alpha$

Proof: The proof is based on the Taylor's expansion of the elements appearing in the iterative expression (2). By using Taylor's expansion around $\alpha$ and using the fact that $\alpha$ is a simple root of $f$, we have

$$
\begin{aligned}
f\left(x_{k}\right) & =f(\alpha)+f^{\prime}(\alpha)\left(x_{k}-\alpha\right)+\frac{1}{2 !} f^{\prime \prime}(\alpha)\left(x_{k}-\alpha\right)^{2}+\frac{1}{3 !} f^{\prime \prime \prime}(\alpha)\left(x_{k}-\alpha\right)^{3}+O\left(e_{k}^{4}\right) \\
& =f^{\prime}(\alpha)\left[e_{k}+c_{2} e_{k}^{2}+c_{3} e_{k}^{3}\right]+O\left(e_{k}^{4}\right)
\end{aligned}
$$

and

$$
f^{\prime}\left(x_{k}\right)=f^{\prime}(\alpha)\left[1+2 c_{2} e_{k}+3 c_{3} e_{k}^{2}\right]+O\left(e_{k}^{3}\right)
$$


By substituting these expressions in the first step of (2), we obtain

$$
y_{k}-\alpha=e_{k}-\varphi \frac{f\left(x_{k}\right)}{f^{\prime}\left(x_{k}\right)}=(1-\varphi) e_{k}+\varphi c_{2} e_{k}^{2}+O\left(e_{k}^{3}\right) .
$$

Expanding in Taylor's series $f^{\prime}\left(y_{k}\right)$ around $\alpha$,

$$
f^{\prime}\left(y_{k}\right)=f^{\prime}(\alpha)\left[1+2 c_{2}\left(y_{k}-\alpha\right)+3 c_{3}\left(y_{k}-\alpha\right)^{2}\right]+O\left(\left(y_{k}-\alpha\right)^{3}\right),
$$

and replacing in (3) the powers of $y_{k}-\alpha$, we get (after some algebraic operations)

$$
f^{\prime}\left(y_{k}\right)=f^{\prime}(\alpha)\left[1-2 c_{2}(\varphi-1) e_{k}+\left(2 \varphi c_{2}^{2}+3 c_{3}(\varphi-1)^{2}\right) e_{k}^{2}\right]+O\left(e_{k}^{3}\right) .
$$

Finally, the error equation is expressed as

$$
\begin{aligned}
e_{k+1}= & (1-\gamma-\delta) e_{k}+(\gamma+\delta-2 \varphi \gamma) c_{2} e_{k}^{2} \\
& +\left[\left(8 \varphi \gamma-2 \gamma-2 \delta-4 \varphi^{2} \gamma\right) c_{2}^{2}+\left(2 \gamma+2 \delta-6 \varphi \gamma+3 \varphi^{2} \gamma\right) c_{3}\right] e_{k}^{3}+O\left(e_{k}^{4}\right)
\end{aligned}
$$

If we force that the terms corresponding to $e_{k}$ and $e_{k}^{2}$ to be eliminated, we have the following system:

$$
\left.\begin{array}{r}
1-\gamma-\delta=0 \\
\gamma+\delta-2 \varphi \gamma=0,
\end{array}\right\}
$$

whose solutions are $\varphi=\frac{1}{2 \gamma}, \quad \delta=1-\gamma$, for all $\gamma \neq 0$, depending on the free parameter $\gamma$. If we replace $\varphi$ and $\delta$ in (2), we have a third-order family whose error equation can be expressed as:

$$
e_{k+1}=\left[\left(8 \gamma-3-4 \gamma^{2}\right) c_{2}^{2}+\left(\frac{11}{4}-6 \gamma+3 \gamma^{2}\right) c_{3}\right] e_{k}^{3}+O\left(e_{k}^{4}\right)
$$

and the theorem is proved.

In order to compare iterative methods it usual to pay attention, not only on the order of convergence, but also on the efficiency of proposed methods. The concept of efficiency index is due to Ostrowski [25], and it is defined as $I=p^{\frac{1}{d}}$, where $p$ is the order of convergence and $d$ is the number of functional evaluations needed per iteration. Then, the efficiency index of the proposed third-order family (including Homeier's method) is $3^{\frac{1}{3}}$, that is always greater than the efficiency index of Newton, $2^{\frac{1}{2}}$.

On the other hand, iterative schemes can also be compared in terms of the asymptotic constant error appearing in the error equation obtained in the proof of Theorem 1. Let us observe that the expression of the error equation depends on parameter $\gamma$. It is easy to observe that there is no value of $\gamma$ that make null simultaneously polynomials $8 \gamma-3-4 \gamma^{2}$ and $\frac{11}{4}-6 \gamma+3 \gamma^{2}$, so there are no method in this family that reaches fourth-order of convergence. However, $\gamma=\frac{1}{2}$ and $\gamma=\frac{3}{2}$ make zero the coefficient of $c_{2}^{2}$, meanwhile $\gamma=\frac{1}{6}(6 \pm \sqrt{3})$ are the roots of the polynomial that is coefficient of $c_{3}$ in the error equation. So, these values of $\gamma$ make the asymptotic error decrease.

In spite of numerous elements of this family have the same order of convergence and efficiency index, not all of them have the same stability properties. This is the reason why we analyze in the next section which members of the class have better behavior, in terms of the set of initial estimations that assure convergence to the roots or values of the parameter with attracting elements that are not solution of the problem to be solved, obtaining a wide range of values of the parameter (that is, members of the class), with excellent stability properties.

\section{Dynamical study}

The application of iterative methods on polynomials gives rise to rational functions whose complex dynamics is not well-known, except the case of Newton's method (see, for example, [7]). From the numerical point of view, the dynamical behavior of the rational function associated with an iterative method gives us important information about its stability and reliability. In this sense, Varona in [29] and Amat et al. in [3] described the dynamical behavior of several well-known families of iterative methods. More recently, in [1, 2, 10, 12, 14, 17, 24, 27], the authors analyze, by using tools of complex dynamics, the qualitative behavior of different known iterative 
methods or families, such as King', Chebyshev-Halley's, c-family or damped Newton. When these kind of analysis is made, different pathological numerical behavior appear, such as periodic orbits, attracting fixed points different from the solution of the problem, etc. A very useful tool to understand the behavior of the different members of the family of methods is the parameter plane, that helps us to select the most stable members of the class.

We study the general convergence of family (2) on quadratic polynomials. To get this aim, we recall some

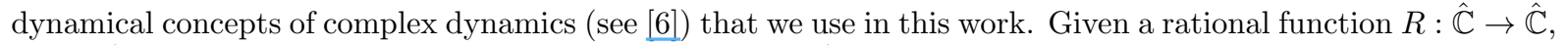
where $\hat{\mathbb{C}}$ is the Riemann sphere, the orbit of a point $z_{0} \in \hat{\mathbb{C}}$ is defined as:

$$
\left\{z_{0}, R\left(z_{0}\right), R^{2}\left(z_{0}\right), \ldots, R^{n}\left(z_{0}\right), \ldots\right\} \text {. }
$$

We analyze the phase plane of the map $R$ by classifying the starting points from the asymptotic behavior of their orbits. A $z_{0} \in \hat{\mathbb{C}}$ is called a fixed point if $R\left(z_{0}\right)=z_{0}$. A periodic point $z_{0}$ of period $p>1$ is a point such that $R^{p}\left(z_{0}\right)=z_{0}$ and $R^{k}\left(z_{0}\right) \neq z_{0}$, for $k<p$. A pre-periodic point is a point $z_{0}$ that is not periodic but there exists a $k>0$ such that $R^{k}\left(z_{0}\right)$ is periodic. A critical point $z_{0}$ is a point where the derivative of the rational function vanishes, $R^{\prime}\left(z_{0}\right)=0$. Moreover, a fixed point $z_{0}$ is called attractor if $\left|R^{\prime}\left(z_{0}\right)\right|<1$, superattractor if $\left|R^{\prime}\left(z_{0}\right)\right|=0$, repulsor if $\left|R^{\prime}\left(z_{0}\right)\right|>1$ and parabolic if $\left|R^{\prime}\left(z_{0}\right)\right|=1$.

The basin of attraction of an attractor $\alpha$ is defined as:

$$
\mathcal{A}(\alpha)=\left\{z_{0} \in \hat{\mathbb{C}}: R^{n}\left(z_{0}\right) \rightarrow \alpha, n \rightarrow \infty\right\} .
$$

The Fatou set of the rational function $R, \mathcal{F}(R)$, is the set of points $z \in \hat{\mathbb{C}}$ whose orbits tend to an attractor (fixed point, periodic orbit or infinity). Its complement in $\hat{\mathbb{C}}$ is the Julia set, $\mathcal{J}(R)$. That means that the basin of attraction of any fixed point belongs to the Fatou set and the boundaries of these basins of attraction belong to the Julia set.

Moreover, it is possible to analyze the behavior of an iterative method acting on any quadratic polynomial.

Definition 1 Let $f$ and $g$ be functions defined as $f, g: \hat{\mathbb{C}} \rightarrow \hat{\mathbb{C}}$. An analytic conjugation between $f$ and $g$ is a diffeomorphism $h: \hat{\mathbb{C}} \rightarrow \widehat{\mathbb{C}}$ such that $h \circ f=g \circ h$.

In the following result it is shown that the rational function associated to the members of the class (2) acting on a polynomial can be transformed by an affine map with no qualitative changes on the dynamics of the family.

Theorem 2 (Scaling Theorem) Let $A(z)=a z+b$ be an affine transformation in $\mathbb{R}$. Let also $f(x)$ be a real function and $g(z)=\lambda(f \circ A)(z)$. Then, the associated operators to family $(2) T_{f}$ and $T_{g}$ are affinely conjugated by $A$. That is, $\left(A \circ T_{g} \circ A^{-1}\right)(z)=T_{f}(z)$, for all $z \in \mathbb{R}$.

Proof: We will prove the equivalent equality:

$$
\left(A \circ T_{g}\right)(z)=\left(T_{f} \circ A\right)(z), \forall z \in \mathbb{R} .
$$

By developing the right side,

$$
\begin{aligned}
\left(T_{f} \circ A\right)(z) & =T_{f}(A(z)) \\
& =A(z)-\left[\gamma \frac{f(A(z))}{f^{\prime}\left(A(z)-\frac{1}{2 \gamma} \frac{f(A(z))}{f^{\prime}(A(z))}\right)}+(1-\gamma) \frac{f(A(z))}{f^{\prime}(A(z))}\right] .
\end{aligned}
$$

On the other hand, by using $A(u-v)=A(u)-A(v)+b$ and $A(c u)=c a u+b$, it can be checked that

$$
\left(A \circ T_{g}\right)(x)=a T_{g}(x)+b=\left(T_{f} \circ A\right)(z)
$$

So, we can use the arbitrary quadratic polynomial $p(z)=(z-a)(z-b)$. For $p(z)$, the operator of the family is the rational function:

$$
T_{p}(z, \gamma, a, b)=\frac{(\gamma-1)(a-z)(z-b)}{a+b-2 z}+\frac{\gamma(z-a)(z-b)}{-2\left(\frac{(a-z)(b-z)}{2 \gamma(a+b-2 z)}+z\right)+a+b}+z,
$$


depending on the parameters $\gamma, a$ and $b$.

Blanchard in [7] considered the conjugacy map $h(z)=\frac{z-a}{z-b}$, (a Möbius transformation) with the following properties:
i) $h(\infty)=1$,
ii) $h(a)=0$,
iii) $h(b)=\infty$,

and proved that, for quadratic polynomials, Newton's operator is conjugate to the rational map $z^{2}$. In an analogous way, operator $T_{p}(z, \gamma, a, b)$ on quadratic polynomials is conjugated to operator $O_{p}(z, \gamma)$,

$$
O_{p}(z, \gamma)=\left(h \circ T_{p} \circ h^{-1}\right)(z)=\frac{z^{3}(\gamma(z+2)-1)}{\gamma+(2 \gamma-1) z} .
$$

We observe that the parameters $a$ and $b$ have been obviated in $O_{p}(z, \gamma)$.

In the following, we calculate the fixed points of $O_{p}(z, \gamma)$ and calculate their stability. On the other hand, the knowledge about the existence of critical points different from the roots of $p(z)$ (that will be called free critical points), is a key fact in the understanding of the global behavior of the family, as establishes the classical result of Fatou and Julia.

Theorem 3 (Fatou [15], Julia [21]) Let $R$ be a rational function. The connected component of the basin containing any attracting periodic point holds, at least, a critical point.

\subsection{Analysis of the fixed and critical points}

Fixed points of $O_{p}(z, \gamma)$ are the roots of the equation $O_{p}(z, \gamma)=z$, that is, $z=0, z=\infty$ (which correspond to the roots $a$ and $b$ of $p(z)$ by Möbius transformation) and, for $\gamma \neq 0$, the following points, that will be called strange fixed points

- $s_{1}(\gamma)=1$,

- $s_{2}(\gamma)=\frac{1}{2 \gamma}\left(-\sqrt{5 \gamma^{2}-6 \gamma+1}-3 \gamma+1\right)$,

- $s_{3}(\gamma)=\frac{1}{2 \gamma}\left(\sqrt{5 \gamma^{2}-6 \gamma+1}-3 \gamma+1\right)$.

Some relations between the strange fixed points are described in the following result.

Lemma 1 The number of simple strange fixed points of operator $O_{p}(z, \gamma)$ is three, except in cases:

i) If $\gamma=1$, then the operator's expression is $O_{p}(z, 1)=z^{3}$, so the only strange fixed point are $z= \pm 1$.

ii) If $\gamma=\frac{1}{3}$, then the operator's expression is $O_{p}\left(x, \frac{1}{3}\right)=-z^{3}$, so the only strange fixed points are $z= \pm i$.

iii) If $\gamma=\frac{1}{5}$, then $s_{1}\left(\frac{1}{5}\right)=s_{2}\left(\frac{1}{5}\right)=s_{3}\left(\frac{1}{5}\right)=1$, as $O_{p}\left(z, \frac{1}{5}\right)=-\frac{(z-3) z^{3}}{3 z-1}$.

Let us notice that for the values of the parameter $\gamma=1$ and $\gamma=\frac{1}{3}$, the method satisfies Cayley's test [4]. So, it can be checked that the existing strange fixed point are always repulsive. Under this circumstances, the behavior of these schemes on quadratic polynomials is like the one of Newton's, but with third order of convergence.

In order to determine the critical points, we calculate the first derivative of $O_{p}(z, \gamma)$,

$$
O_{p}^{\prime}(z, \gamma)=\frac{z^{2}\left(6 \gamma^{2}(z+1)^{2}-\gamma(z(3 z+8)+3)+2 z\right)}{(\gamma+(2 \gamma-1) z)^{2}} .
$$

As we have mentioned previously in Theorem 3, there is at least one critical point associated with each basin of attraction. It is clear that $z=0$ and $z=\infty$ (related to the roots of the polynomial by means of Möbius map) are critical points and give rise to their respective Fatou components, but there exist in the family other free critical points, depending on the value of the parameter.

Lemma 2 Analyzing the equation $O_{p}^{\prime}(z, \gamma)=0$, we obtain that

a) If $\gamma=1$ or $\gamma=\frac{1}{3}$, there are no free critical points of operator $O_{p}(z, \gamma)$. 
b) In any other case,

$$
c r_{1}(\gamma)=\frac{6 \gamma^{2}+\sqrt{-12 \gamma^{3}+19 \gamma^{2}-8 \gamma+1}-4 \gamma+1}{3 \gamma-6 \gamma^{2}} \quad \text { and } \quad c r_{2}(\gamma)=\frac{6 \gamma^{2}-\sqrt{-12 \gamma^{3}+19 \gamma^{2}-8 \gamma+1}-4 \gamma+1}{3 \gamma-6 \gamma^{2}}=\frac{1}{c r_{1}}
$$

are free critical points.

From the previous results, let us summarize:

- There are two dependent free critical points, $c r_{1}(\gamma)=\frac{1}{c r_{2}(\gamma)}$. So, we will consider only $c r_{1}(\gamma)$.

- If $\gamma=\frac{1}{5}$, then the associated operator is $O_{p}\left(z, \frac{1}{5}\right)=-\frac{(z-3) z^{3}}{3 z-1}$ and there are is one strange fixed point, $z=1$.

- When $\gamma=1$ or $\gamma=\frac{1}{3}$, the associated operator is $O_{p}(z, 1)=z^{3}$ and $O_{p}\left(z, \frac{1}{3}\right)=-z^{3}$ respectively, there are only repulsive strange fixed points and there are not free critical points.

As we will see in the following section, not only the number but also the stability of the fixed points depend on the parameter of the family. The relevance of this study yields in the fact that the existence of attracting strange fixed points can make the iterative scheme converge to a "false" solution.

\subsection{Stability of the fixed points}

As the order of convergence of the family is three, it is clear that the origin and $\infty$ are always superattractive fixed points, but the stability of the other fixed points gives us interesting numerical information. In the following results we show the stability of the strange fixed points.

Theorem 4 The character of the strange fixed point $s_{1}(\gamma)=1$ is as follows:

i) If $\left|\gamma-\frac{26}{110}\right|<\frac{2}{55}$, then $s_{1}(\gamma)=1$ is an attractor, and superattractor when $\gamma=\frac{1}{4}$.

ii) When $\left|\gamma-\frac{26}{110}\right|=\frac{2}{55}, s_{1}(\gamma)=1$ is a parabolic point.

iii) If $\left|\gamma-\frac{26}{110}\right|>\frac{2}{55}$, then $s_{1}(\gamma)=1$ is a repulsor.

Proof: It is easy to check that

$$
O_{p}^{\prime}(1, \gamma)=\frac{2-8 \gamma}{1-3 \gamma}
$$

So,

$$
\left|\frac{2-8 \gamma}{1-3 \gamma}\right|<1 \quad \text { is equivalent to } \quad|2-8 \gamma|<|1-3 \gamma| .
$$

Let us consider $\gamma=a+i b$ an arbitrary complex number. Then,

$$
4+64 a^{2}-32 a+64 b^{2}<1+9 a^{2}-6 a+9 b^{2} .
$$

By simplifying

$$
3+55 a^{2}-26 a+55 b^{2}<0
$$

that is,

$$
\left(a-\frac{26}{110}\right)^{2}+b^{2}<\left(\frac{2}{55}\right)^{2}
$$

Therefore,

$$
\left|O_{\gamma}^{\prime}(1)\right|<1 \quad \text { if and only if } \quad\left|\gamma-\frac{26}{110}\right|<\frac{2}{55} .
$$

Finally, if $\gamma$ satisfies $\left|\gamma-\frac{26}{110}\right|>\frac{2}{55}$, then $\left|O_{p}^{\prime}(1, \gamma)\right|>1$ and $z=1$ is a repulsive point.

A similar result can be proved for the rest of strange fixed points. 
Theorem 5 The analysis of the stability of strange points $s_{2}(\gamma)$ and $s_{3}(\gamma)$ shows that:

i) If $\left|\gamma-\frac{12}{70}\right|<\frac{1}{35}$, then both points are attractors and they are superattractors when $\gamma=\frac{1}{6}$.

ii) If $\left|\gamma-\frac{12}{70}\right|=\frac{1}{35}$, then $s_{2}(\gamma)$ and $s_{3}(\gamma)$ are parabolic.

iii) In any other case, both are repulsors.

The proof of this theorem is analogous to that of Theorem 4 , by using the stability function of $s_{2}(\gamma)$ and $s_{3}(\gamma)$,

$$
O_{p}^{\prime}\left(\frac{-\sqrt{5 \gamma^{2}-6 \gamma+1}-3 \gamma+1}{2 \gamma}, \gamma\right)=O_{p}^{\prime}\left(\frac{\sqrt{5 \gamma^{2}-6 \gamma+1}-3 \gamma+1}{2 \gamma}, \gamma\right)=6-\frac{1}{\gamma}
$$

In Figures 1 and 2, we represent the stability regions $\left|O_{p}^{\prime}\left(s_{i}(\gamma), \gamma\right)\right|$ of each strange fixed point $s_{i}(\gamma), i=1,2,3$, that we get from Theorem 4 and Theorem 5 . These regions are represented by cones whose base is formed by all the complex values of $\gamma$ that make the strange fixed point attracting and whose vertices correspond to those values of $\gamma$ that minimize the stability function. If the stability function is null at these points, then the strange fixed point under study is superattracting.

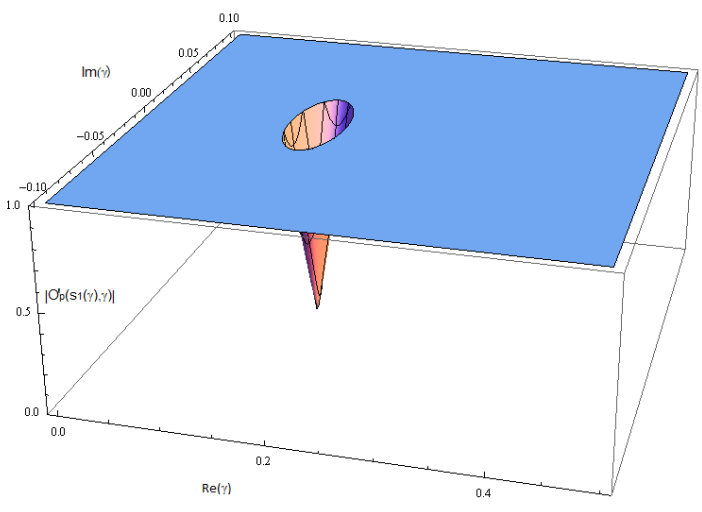

Figure 1: Stability region of $s_{1}(\gamma)$.

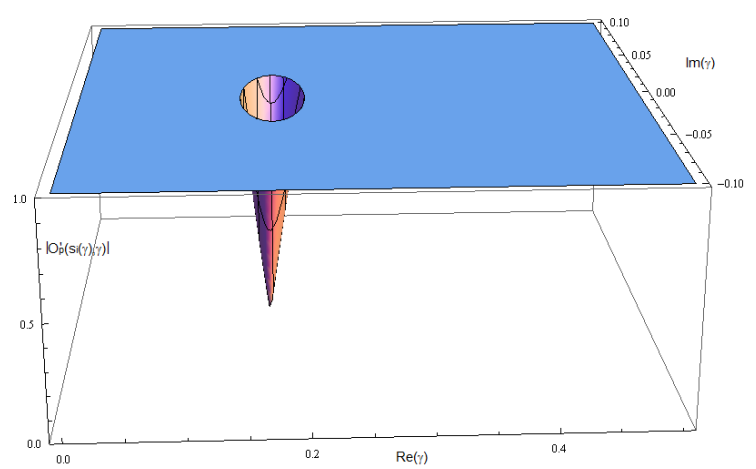

Figure 2: Stability region of $s_{i}(\gamma), i=2,3$.

\subsection{The parameter space}

As we have seen, the dynamical behavior of operator $O_{p}(z, \gamma)$ depends on the values of parameter $\gamma$. The parameter space associated with a free critical point of operator (7) is obtained by associating each point of the parameter plane with a complex value of $\gamma$, i.e., with an element of family (2). Every value of $\gamma$ belonging to the same connected component of the parameter space gives rise to subsets of schemes of family (2) with similar dynamical behavior. So, it is interesting to find regions of the parameter plane as much stable as possible, because these values of $\gamma$ will give us the best members of the family in terms of numerical stability.

As $c r_{1}(\gamma)=\frac{1}{c r_{2}(\gamma)}$, we have one free independent critical point, so we obtain one parameter plane, that we call $P_{1}$. When we consider the free critical point $z=c r_{1}(\gamma)$, as a starting point of the iterative scheme of the family associated to each complex value of $\gamma$, we paint this point of the complex plane in red if the method converges to any of the roots (zero and infinity) and they are black in other cases. The color used is brighter when the number of iterations is lower. The parameter plane appearing in Figure 3a has been generated by using the routines described in [10]. A mesh of $2000 \times 2000$ points has been used, 1000 has been the maximum number of iterations involved and $10^{-3}$ the tolerance used as a stopping criterium. Let us observe a vertical structure that contains (in its middle) the detail appearing in Figure 3b. The other black regions appearing in this structure are Mandelbrot sets and correspond to different periodic behaviors.

We can observe (in Figure 3b) two biggest black disks on the centre of the figure: it is the region where strange fixed points $s_{2,3}(\gamma)$ (the disk on the left) and $s_{1}(\gamma)$ (the disk on the right) are attractive or superattractive (see Theorem 5). 


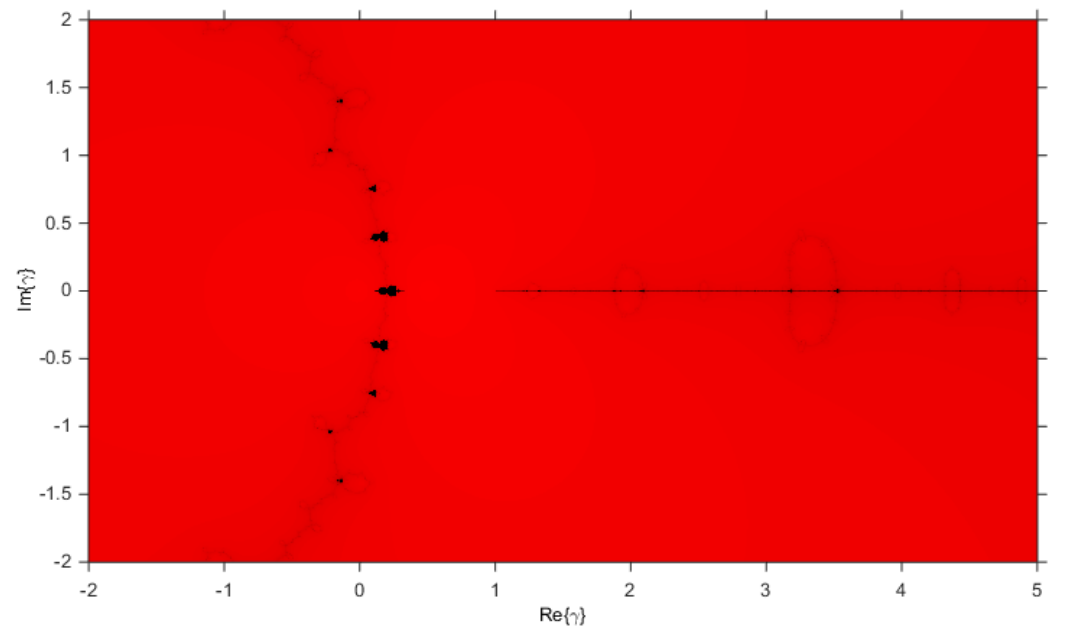

(a) Parameter plane $P_{1}$

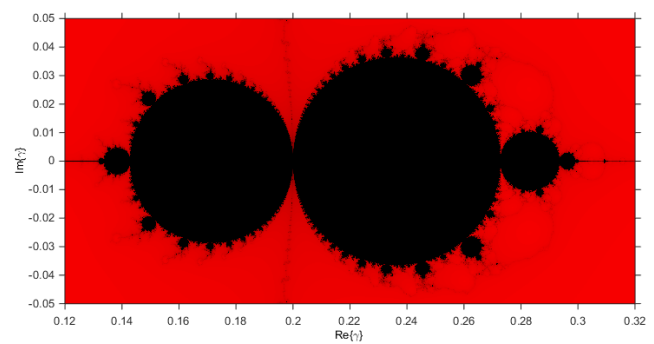

(b) A detail

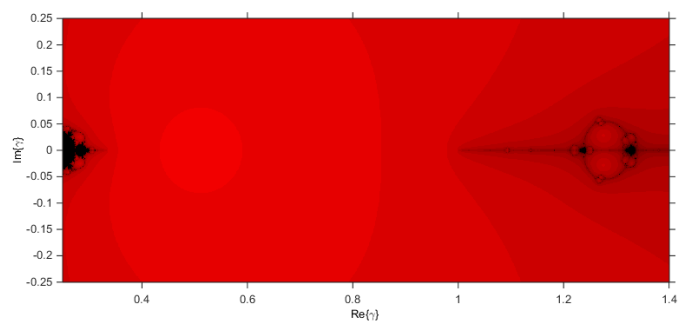

(c) A detail

Figure 3: Parameter plane $P_{1}$ and two details

In Figure 3b, at the right of the biggest black disk we see a smaller one, which we are going to show now that it corresponds to values of $\gamma$ for which there are also attractive orbits of period 2. Also, in Figure $3 \mathrm{c}$ the region near zero is shown, where both antennas are visible. Let us remark that, in these antennas, some Mandelbrot sets appear showing periodic behaviors.

Summarizing, it is observed that the most of the complex plane correspond to values of the parameter with stable behavior, meanwhile pathological cases appear in very small regions.

\subsection{Orbits of period two}

In order to obtain the analytical expression of the elements of 2-periodic orbits, depending on $\gamma$, we calculate $O_{p}\left(O_{p}(z, \gamma)\right)$, that will be denoted by $O_{p}^{2}(z, \gamma)$

$$
O_{p}^{2}(z, \gamma)=\frac{z^{9}(\gamma(z+2)-1)^{3}\left(-\gamma\left(z^{3}+4 z+1\right)+\gamma^{2}\left(z^{4}+2 z^{3}+4 z+2\right)+z\right)}{(\gamma+2 \gamma z-z)^{3}\left(z^{3}+\gamma^{2}\left(2\left(z^{4}+2 z^{3}+z\right)+1\right)-\gamma\left(z^{4}+4 z^{3}+z\right)\right)}
$$


The periodic points of $O_{p}(z, \gamma)$ with period two are the roots of the equation $O_{p}^{2}(z, \gamma)=z$, that is, the fixed points, the periodic points

$$
\begin{aligned}
& p e_{\{1,2\}}(\gamma)=-\frac{\sqrt{5 \gamma^{2}-2 \gamma+1}}{4 \gamma} \pm \frac{1}{2} \sqrt{\frac{(3 \gamma-1)^{2}}{2 \gamma^{2}}-\frac{\gamma\left(-\frac{(3 \gamma-1)^{3}}{\gamma^{3}}+\frac{4(3 \gamma-1)^{2}}{\gamma^{2}}-\frac{8(3 \gamma-1)}{\gamma}\right)}{2 \sqrt{5 \gamma^{2}-2 \gamma+1}}-\frac{3 \gamma-1}{\gamma}-2}-\frac{3 \gamma-1}{4 \gamma}, \\
& p e_{\{3,4\}}(\gamma)=\frac{\sqrt{5 \gamma^{2}-2 \gamma+1}}{4 \gamma} \pm \frac{1}{2} \sqrt{\frac{(3 \gamma-1)^{2}}{2 \gamma^{2}}+\frac{\gamma\left(-\frac{(3 \gamma-1)^{3}}{\gamma^{3}}+\frac{4(3 \gamma-1)^{2}}{\gamma^{2}}-\frac{8(3 \gamma-1)}{\gamma}\right)}{2 \sqrt{5 \gamma^{2}-2 \gamma+1}}-\frac{3 \gamma-1}{\gamma}-2}-\frac{3 \gamma-1}{4 \gamma}
\end{aligned}
$$

and also the roots of several polynomial whose analytical expression have not been obtained.

In Figure 4, we present the stability regions of $p e_{i}(\gamma), i=1,2$, and in Figure 5, we present the stability regions of $p e_{i}(\gamma), i=3,4$. They represent the complex area where $\left|O_{p}^{\prime 2}\left(p e_{1}(\gamma), \gamma\right) \cdot O_{p}^{\prime 2}\left(p e_{2}(\gamma), \gamma\right)\right|<1$ or $\left|O_{p}^{\prime 2}\left(p e_{3}(\gamma), \gamma\right) \cdot O_{p}^{\prime 2}\left(p e_{4}(\gamma), \gamma\right)\right|<1$. In Figure 6 we show all stability regions that can be compared with the parameter plane in Figure $3 \mathrm{a}$.

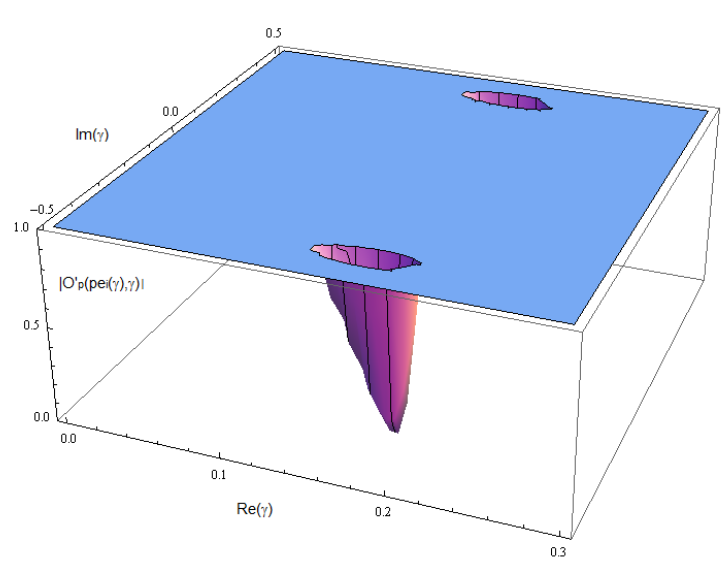

Figure 4: Stability regions of $p e_{i}(\gamma), i=1,2$

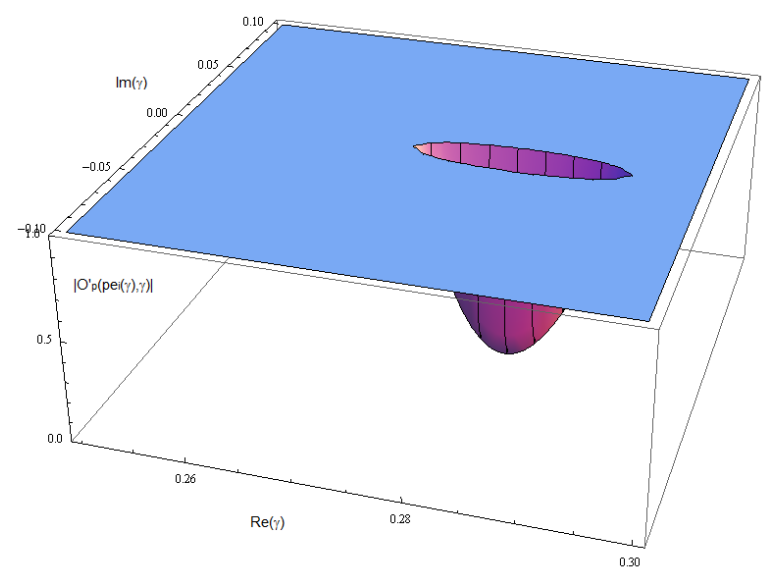

Figure 5: Stability regions of $p e_{i}(\gamma), i=3,4$

Moreover, it can be checked that there exist three values of parameter $\gamma$ that make the 2-periodic orbits superattracting, that is, satisfies $O_{p}^{\prime 2}(z, \gamma)=0$. These values are $\gamma=0.19239 \pm i 0.40009$ and $\gamma=0.28187$ and belong to the black cardioids in the big structure of the parameter plane $P_{1}$ and to the bulb on the right of the biggest disk in Figure 3b, respectively.

\subsection{Dynamical Planes}

In this section we will show, by means of dynamical planes, the qualitative behavior of the different elements of family (2). We will select this elements by using the conclusions obtained by analyzing the parameter planes of the family.

As in case of parameter planes, these dynamical planes has been generated by using the routines appearing in [10]. The dynamical plane associated to a value of the parameter $\gamma$, that is, corresponding to an element of family (2), is generated by using each point of the complex plane as initial estimation (we have used a mesh of $400 \times 400$ points). We paint in blue the points whose orbit converges to infinity, in orange the points converging to zero (with a tolerance of $10^{-3}$ ), in green those points whose orbit converges to one of the strange fixed points (all fixed points appear marked as a white star in the figures) and in black if it reaches the maximum number of 40 iterations without converging to any of the fixed points (for interpretation of the references to color in the text, the reader is referred to the web version of this article).

Most part of the regions in parameter space $P_{1}$ correspond to iterative methods with good numerical behavior, in terms of stability and efficiency. They correspond to the values of $\gamma$ painted in red in the parameter plane $P_{1}$. In Figures 7, 8, 9 and 10 we show different stable behavior corresponding to several values of $\gamma$ selected in this red regions; in particular, we use $\gamma=\frac{1}{2}$ (Homeier's method), $\gamma=-0.4, \gamma=0.4$ and $\gamma=-0.08$.

On the other hand, unstable behavior is found when we choose values of $\gamma$ in the black regions of parameter plane $P_{1}$. In Figure 11, the dynamical plane of the iterative method corresponding to $\gamma=0.181$ is presented, 


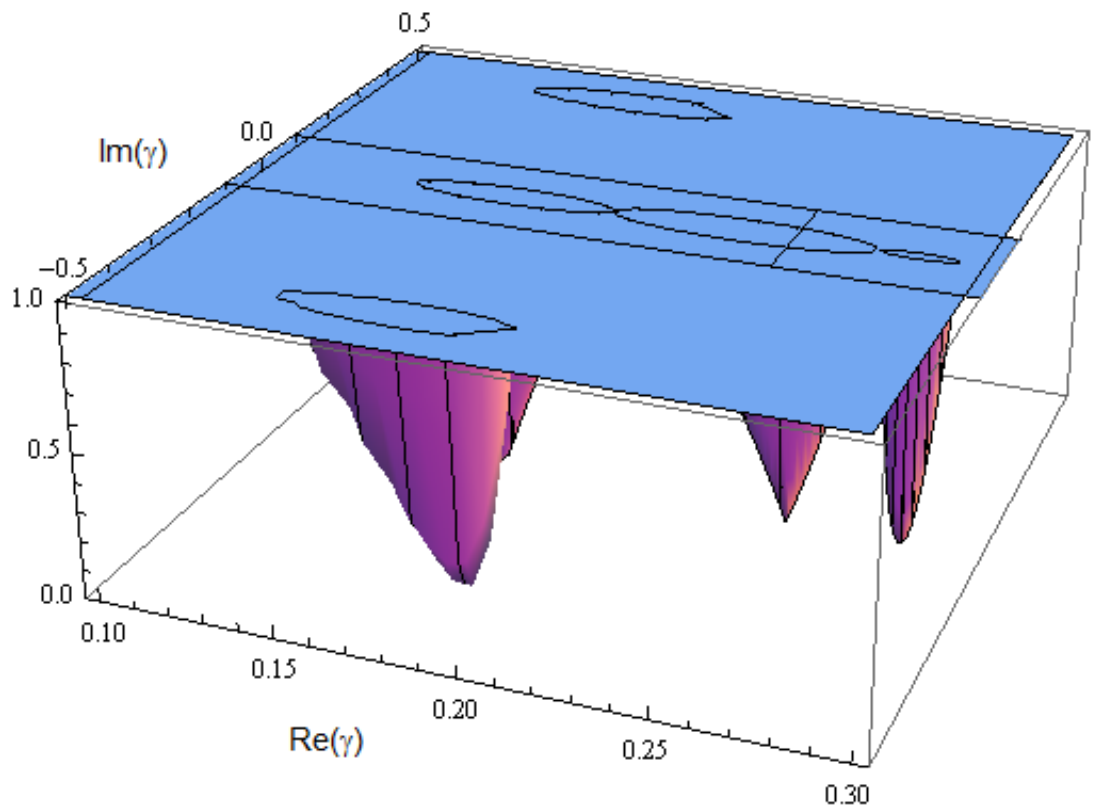

Figure 6: Stability regions (all together)

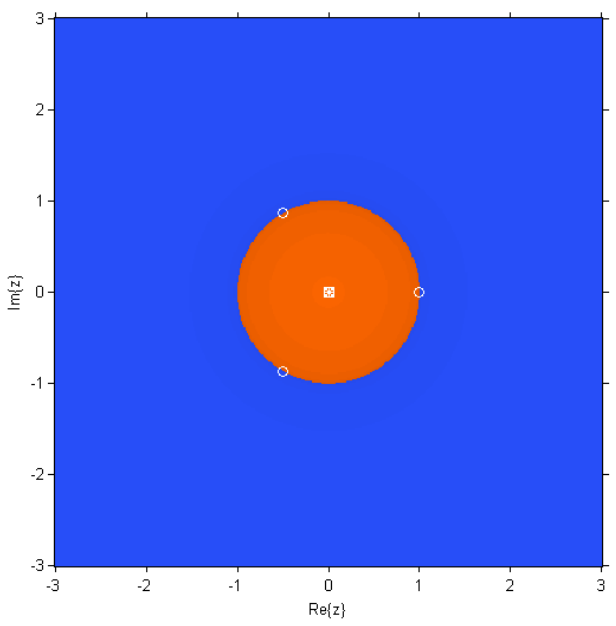

Figure 7: Dynamical plane for $\gamma=0.5$

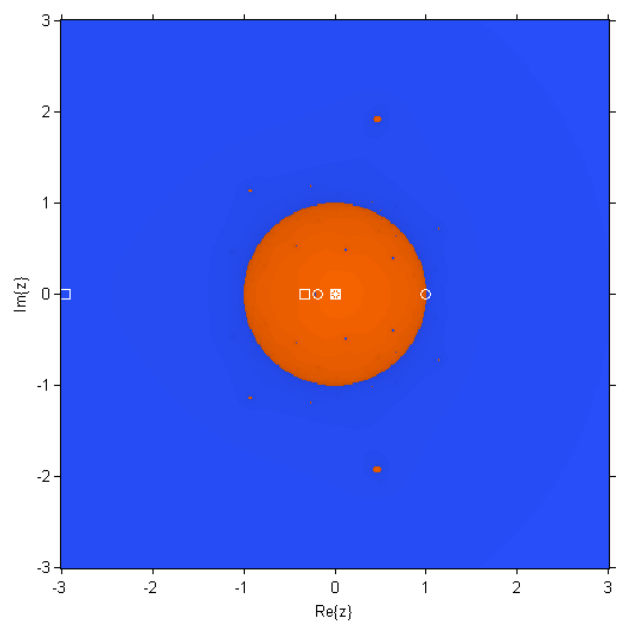

Figure 8: Dynamical plane for $\gamma=-0.4$

showing the existence of four different basins of attraction, two of them of the superattractors 0 and $\infty$ and the other two corresponding to the attractors $s_{2}(\gamma)$ and $s_{3}(\gamma)$. Analogously, in Figure 12 we see, besides the 0 and $\infty$ basins of attraction, the one corresponding to the attractor $s_{1}(\gamma)$.

Figure 13 corresponds to the dynamical plane for $\gamma=0.1752+0.4 i$, showing the existence of three different basins of attraction, two of them of the superattractors 0 and $\infty$ and the other one corresponding to an attractive orbit of period 2 .

Other regions of the parameter plane $P_{1}$ where there are attractive orbits of period 2 are the ones corresponding to Figure 14 and 15. Let's notice that in this last one (Figure 15) there are two different orbits of period 2 with a free critical point contained in each one of their basins of attraction. A 4-period orbit is found in the dynamical plane corresponding to $\gamma=1.24$, as it is shown in Figure 16, that corresponds to values of $\gamma$ in one of the Mandelbrot sets in the left of antenna of the parameter plane (see Figure 3c). 


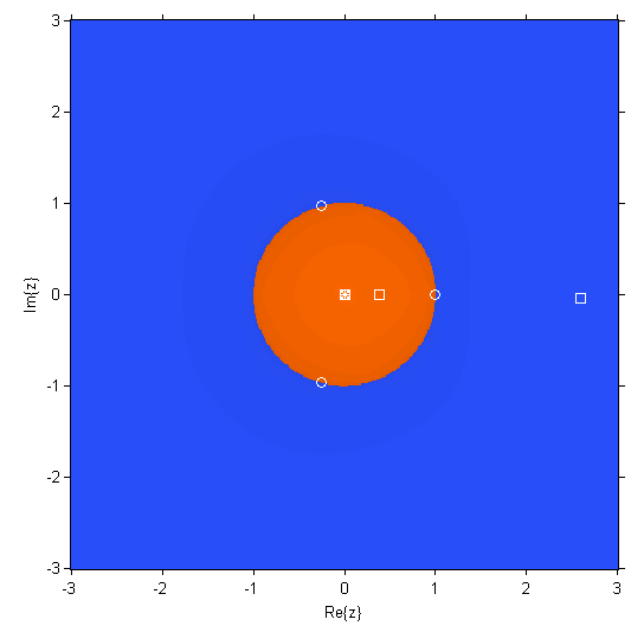

Figure 9: Dynamical plane for $\gamma=0.4$

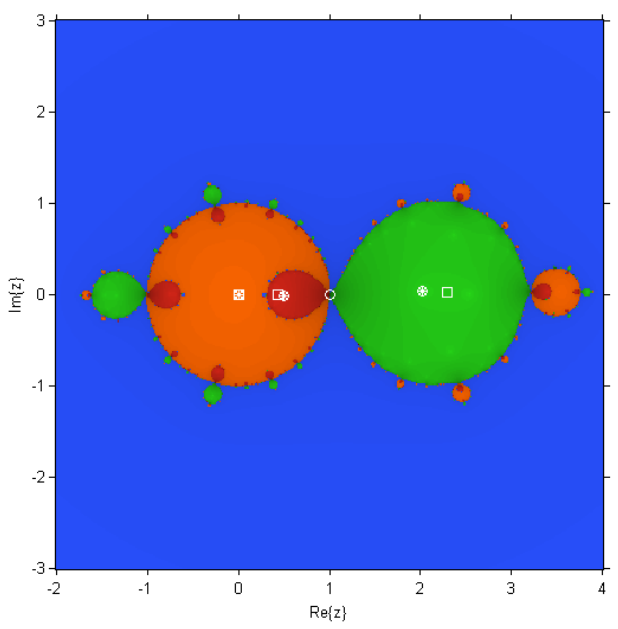

Figure 11: Dynamical plane for $\gamma=0.181$

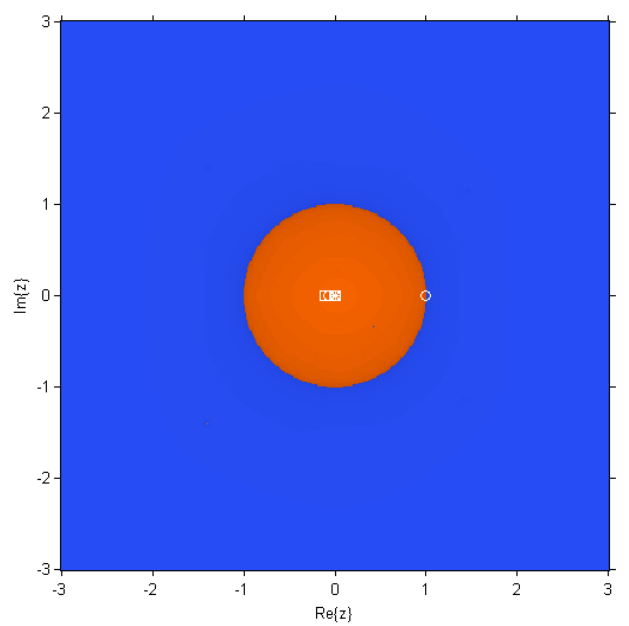

Figure 10: Dynamical plane for $\gamma=-0.08$

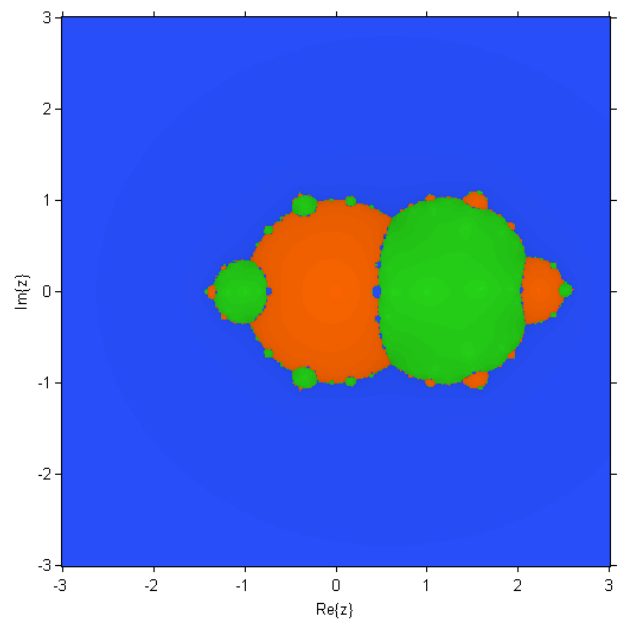

Figure 12: Dynamical plane for $\gamma=0.23$

Finally, a dynamical plane corresponding to $\gamma=0.31$ is shown in Figure 17 in order to see the behavior of the right of the antenna in $P_{1}$; even this area shows a very good stability of the method.

\section{Application to planar 1D-Bratu problem}

We consider the classical Bratu problem [9] which is an elliptic nonlinear partial differential equation with homogeneous Dirichlet boundary conditions. In the planar 1D-case, the problem can be expressed as

$$
\begin{aligned}
u_{x x}+C e^{u} & =0, \quad 0 \leq x \leq 1, \\
u(0)=u(1) & =0 .
\end{aligned}
$$

This problem appears in a large variety of application areas such as the fuel ignition model of thermal combustion, radiative heat transfer, thermal reaction, the Chandrasekhar model of the expansion of the universe, chemical reactor theory and nanotechnology $[16, \underline{30}, \underline{19}, \underline{20}$. Parameter $C$ involved in Bratu problem has a 


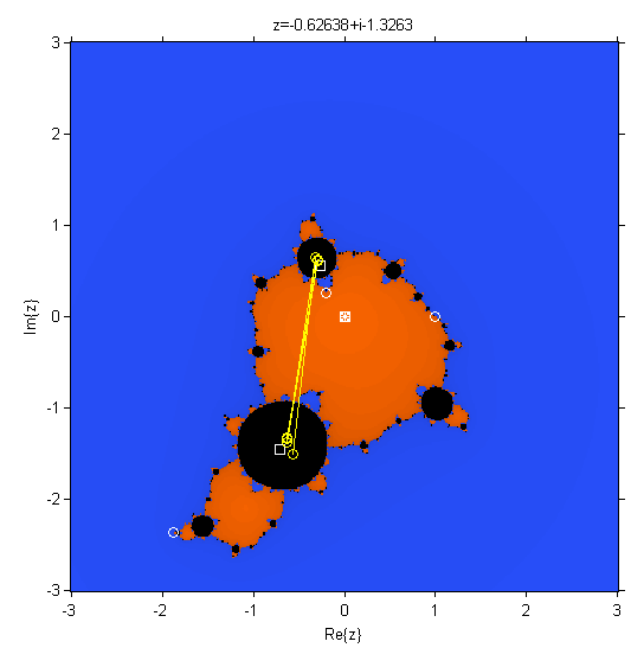

Figure 13: Dynamical plane for $\gamma=0.1752+0.4 i$

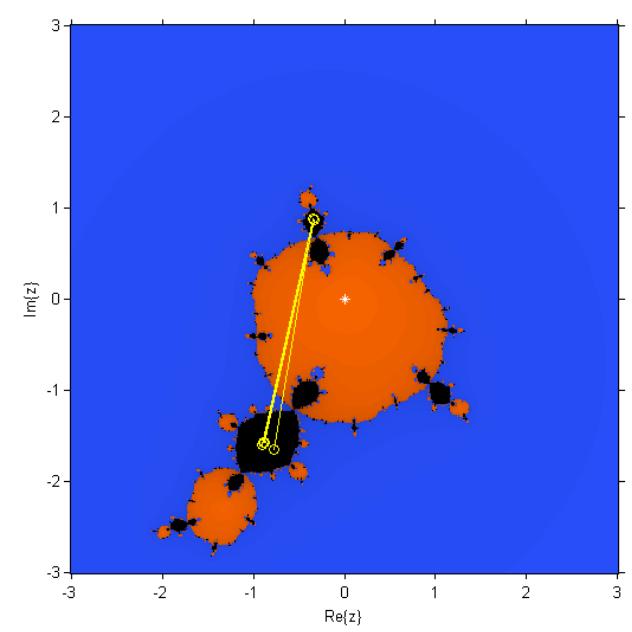

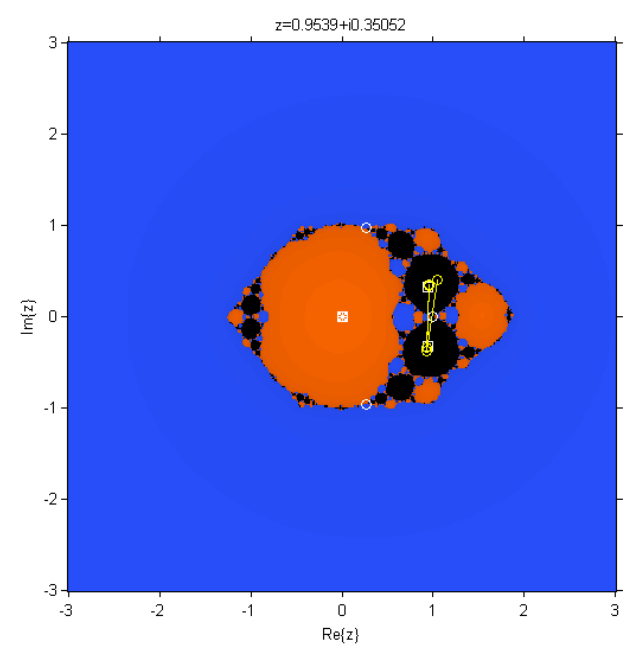

Figure 14: Dynamical plane for $\gamma=0.283$

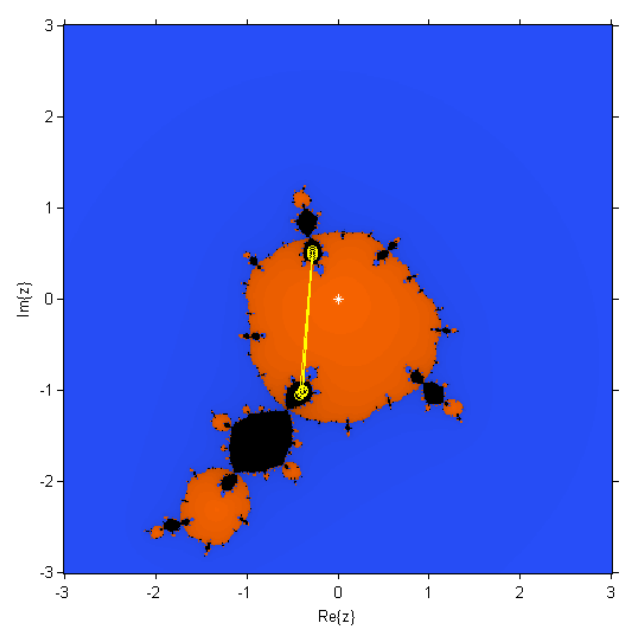

Figure 15: Dynamical planes for $\gamma=0.12+0.4 i$

critical turning point at $C_{c} \approx 3.513830719$. So, Bratu problem has two solutions for $C<C_{c}$, leading to two branches; there is only one solution for $C=C_{c}$ and no solution for $C>C_{c}$.

The exact solution to the planar 1D-Bratu problem is given by:

$$
u(x)=2 \ln \left[\frac{\cosh (\alpha)}{\cosh (\alpha(1-2 x))}\right],
$$

where $\alpha$ satisfies the transcendental equation

$$
\cosh (\alpha)=\frac{4}{\sqrt{2 C}} \alpha .
$$

The solution of this equation may be performed using the classical Newton method, which results can be observed in Table 1, for two values of $C$, one of them stable $C=3$ and also $C=3.51 C=3.5138$ (close to the critical turning value); all of them will be used in the numerical experiments. Let's notice that depending on the initial estimation of the iterative method, either the value corresponding to the lower or upper branch is obtained. 


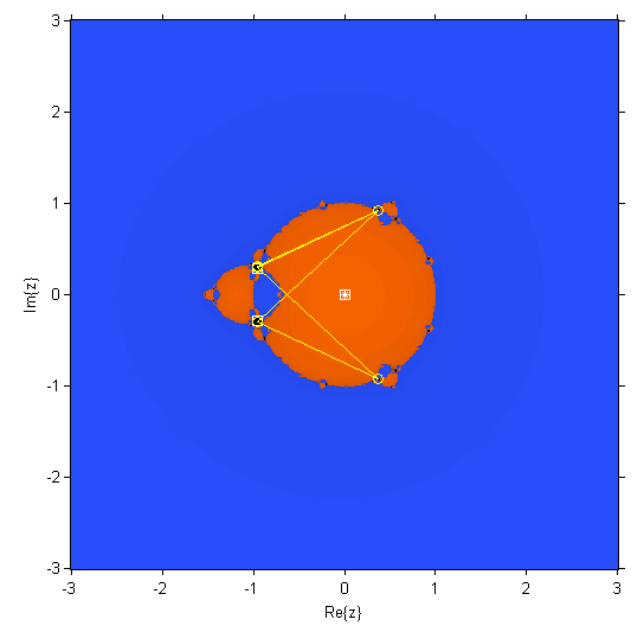

Figure 16: Dynamical plane for $\gamma=1.24$

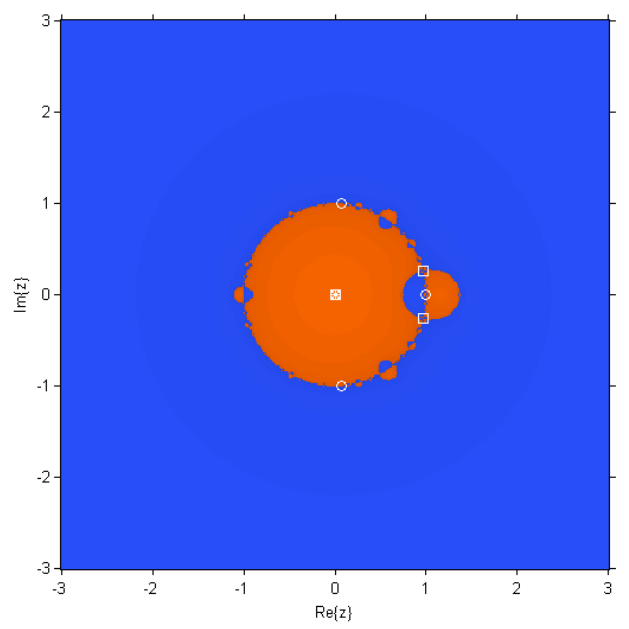

Figure 17: Dynamical plane for $\gamma=0.31$

\begin{tabular}{|c|c|c|}
\cline { 2 - 3 } \multicolumn{1}{c|}{} & \multicolumn{2}{c|}{ Value of $\alpha$} \\
\hline$C$ & Lower branch & Upper branch \\
\hline 3 & 0.8434 & 1.6441 \\
\hline 3.51 & 1.1669 & 1.2330 \\
\hline 3.5138 & 1.1967 & 1.2026 \\
\hline
\end{tabular}

Table 1: Both solutions, lower and upper, of equation (10) for different values of $C$

The solution of the problem using the finite-difference method involves discretizing the differential equation (8), including the boundary conditions. The method transforms the problem into a system of simultaneous nonlinear equations which are then solved by means of different iterative methods of the family (2), that showed stable behavior in the dynamical analysis made in Section 3. Using a uniform grid with separation $h=1 /(n+1)$ for integer $n$, the range $0 \leq x \leq 1$ is discretized as $x_{j}=0+j h$, with $j=0,1, \ldots, n+1$.

Then, a standard second order finite-difference scheme yields the discrete version of the planar Bratu problem,

$$
\frac{u_{i+1}-2 u_{i}+u_{i-1}}{h^{2}}+C e^{u_{i}}=0, \quad i=1,2, \ldots, n,
$$

with $u_{0}=u_{n+1}=0$. In the tests, we take $n=20$.

For treating the resulting nonlinear system, it is well-known that the solution is much more reliable and obtained much more quickly if the initial guess is good. Then, a simple sinusoidal starting function (see [8] and $[23])$, us $(x)=a \sin (\pi x)$, having appropriate amplitude satisfying the boundary conditions, is used. In particular, for the lower branch, $a$ should be taken as $a<u_{c}$, while the upper branch requires $a>u_{c}$ (otherwise, it could happen that due to a wrong initial guess, our method would converge to the opposite solution), where $u_{c}$ is the unique solution of (9) when $C=C_{c}$. We have chosen $a=1$ and $a=3$ respectively. So, for each value of $C$ and $a$, we calculate the estimated solution of the nonlinear system (11) by using several schemes. The stopping criteria used is $\left\|u^{(k+1)}-u^{(k)}\right\|<10^{-25}$ or $\left\|F\left(u^{(k+1)}\right)\right\|<10^{-25}$ and the maximum of iterations considered is 100. The information displayed at Table 2 corresponds to the estimations of the error of the iterative process $\left\|u^{(k+1)}-u^{(k)}\right\|$ and $\left\|F\left(x^{(k+1)}\right)\right\|$ and the number of iterations, for $C=3$, and values $a=1$ and $a=3$, in order to see the performance of the methods approximating each one of the branches of the solution. To verify the theoretical order of convergence $(p)$ of the methods, we calculate the computational order of convergence (ACOC) introduced in $\underline{[13]}$ as

$$
p \approx A C O C=\frac{\ln \left(\left\|x^{(k+1)}-x^{(k)}\right\| /\left\|x^{(k)}-x^{(k-1)}\right\|\right)}{\ln \left(\left\|x^{(k)}-x^{(k-1)}\right\| /\left\|x^{(k-1)}-x^{(k-2)}\right\|\right)} .
$$

The approximated computational order of convergence $A C O C$ is also displayed in Table 2. 


\begin{tabular}{|c|c|c|c|c|c|}
\hline & $\gamma$ & $\left\|u^{(k+1)}-u^{(k)}\right\|$ & $\left\|F\left(u^{(k+1)}\right)\right\|$ & iter & ACOC \\
\hline \hline$C=3$ & 0.5 & $3.24 \mathrm{e}-21$ & $2.40 \mathrm{e}-40$ & 4 & 2.9949 \\
$a=1$ & -0.4 & $6.43 \mathrm{e}-12$ & $4.31 \mathrm{e}-37$ & 5 & 2.9655 \\
& 0.4 & $1.79 \mathrm{e}-16$ & $3.79 \mathrm{e}-40$ & 4 & 2.9927 \\
& -0.08 & $5.65 \mathrm{e}-17$ & $5.35 \mathrm{e}-40$ & 5 & 2.9905 \\
\hline$C=3$ & 0.5 & $1.07 \mathrm{e}-09$ & $3.41 \mathrm{e}-31$ & 4 & 3.0781 \\
$a=3$ & -0.4 & $1.05 \mathrm{e}-09$ & $8.45 \mathrm{e}-30$ & 5 & 2.9467 \\
& 0.4 & $9.48 \mathrm{e}-12$ & $4.17 \mathrm{e}-37$ & 4 & 2.8510 \\
& -0.08 & $4.29 \mathrm{e}-16$ & $8.13 \mathrm{e}-40$ & 6 & 2.9902 \\
\hline \hline
\end{tabular}

Table 2: Approximating results of new and known methods on Bratu problem for $C=3$

Observed values in Table 2 show a stable behavior in all the methods, being the best ones those with $\gamma=0.5$ (Homeier's method) and $\gamma= \pm 0.4$, two of the proposed schemes. All the estimations are close enough to the solution but adding one more iteration does not improve it: the difference between the two last iterations and the norm of the system at the last iteration is in the same range as the rest of the methods, that has one iteration less. Moreover, In Table 3 , as the exact solution $u$ is known, the norm of the exact error $\left\|u-u^{(k+1)}\right\|$ and the maximum error $\max _{i \in\{1, \ldots, n\}}\left|u_{i}-u_{i}^{(k+1)}\right|$ are shown, both for lower (LB) and upper branch (UB). Let us remark that all the displayed errors coincide for the methods under analysis.

\begin{tabular}{|c|c|c|c|c|c|}
\hline & $\gamma$ & $\left\|u-u^{(k+1)}\right\|_{L B}$ & $\max _{i \in 1, \ldots, n}\left|u_{i}-u_{i}^{(k+1)}\right|_{L B}$ & $\left|u-u^{(k+1)}\right|_{U B}$ & $\max _{i \in 1, \ldots, n}\left|u_{i}-u_{i}^{(k+1)}\right|_{U B}$ \\
\hline \hline$C=3$ & 0.5 & 0.0034 & 0.0011 & 4.2157 & 1.3295 \\
$a=1$ & -0.4 & 0.0034 & 0.0011 & 4.2157 & 1.3295 \\
& 0.4 & 0.0034 & 0.0011 & 4.2157 & 1.3295 \\
& -0.08 & 0.0034 & 0.0011 & 4.2157 & 1.3295 \\
\hline$C=3$ & 0.5 & 4.2044 & 1.3265 & 0.0148 & 0.0041 \\
$a=3$ & -0.4 & 4.2044 & 1.3265 & 0.0148 & 0.0041 \\
& 0.4 & 4.2044 & 1.3265 & 0.0148 & 0.0041 \\
& -0.08 & 4.2044 & 1.3265 & 0.0148 & 0.0041 \\
\hline \hline
\end{tabular}

Table 3: Errors of new and known methods on Bratu problem for $C=3$

Results presented in Table 3 are the expected ones: when $a=1$, all the methods converge to the solution at the lower branch, as the exact error at both branches shows. If $a=3$, the lowest error appears at the upper branch. Let us remark that the exact error is much bigger than the tolerance used in the iterative method, this follows from the order of the discretization of the original differential equation. Moreover, all the exact errors coincide, in spite of the different iterative methods used.

However, if $C=3.51$ is considered (see Tables 4 and 5 ), the proximity to the critical value $C_{c}$ yields different performances of the methods. In order to visualize this behavior, higher tolerance of the error has been used, being the stopping criteria used in this case $\left\|u^{(k+1)}-u^{(k)}\right\|<10^{-4}$ or $\left\|F\left(u^{(k+1)}\right)\right\|<10^{-4}$. Let us notice that, due to this new tolerance, the number of iterations needed is very low. Then, the estimated order of convergence ACOC cannot be calculated and it does not appear in Table 4, nor in Table 6.

Although the difference between the two last iterates is quite big, the value of the nonlinear system at the last iterate $\left\|F\left(u^{(k+1)}\right)\right\|$ satisfies the stopping criterium. Moreover, the processes are, in general, more stable to find the solution at the lower branch than the one at the upper one, as can be noticed by the number of iterations needed. The best performance corresponds to one of the new methods, for $\gamma=0.4$. Indeed, the exact error of the iterative schemes are different, as can be observed in Table 5. The performance of the proposed methods is good in all cases, finding the lowest exact error in the results from $\gamma=0.5$ for the lower branch and $\gamma=-0.4$ in case of convergence to upper branch.

Finally, when $C=3.5138$ is considered (see Tables 6 and 7 ), the critical value $C_{c}$ is very close and it makes the process highly unstable. In fact, as can be observed in Table 6, the showed results correspond to the 100th- 


\begin{tabular}{|c|c|c|c|c|}
\hline & $\gamma$ & $\left\|u^{(k+1)}-u^{(k)}\right\|$ & $\left\|F\left(u^{(k+1)}\right)\right\|$ & iter \\
\hline \hline$C=3.51$ & 0.5 & 0.4848 & $6.91 \mathrm{e}-05$ & 1 \\
$a=1$ & -0.4 & 0.1510 & $3.85 \mathrm{e}-05$ & 2 \\
& 0.4 & 0.5414 & $3.20 \mathrm{e}-05$ & 1 \\
& -0.08 & 0.1532 & $5.66 \mathrm{e}-05$ & 2 \\
\hline$C=3.51$ & 0.5 & 0.1544 & $1.09 \mathrm{e}-05$ & 4 \\
$a=3$ & -0.4 & 0.1788 & $5.98 \mathrm{e}-05$ & 5 \\
& 0.4 & 0.3945 & $2.79 \mathrm{e}-05$ & 3 \\
& -0.08 & 0.1269 & $4.34 \mathrm{e}-05$ & 6 \\
\hline \hline
\end{tabular}

Table 4: Approximating results of new and known methods on Bratu problem for $C=3.51$

\begin{tabular}{|c|c|c|c|c|c|}
\hline & $\gamma$ & $\left\|u-u^{(k+1)}\right\|_{L B}$ & $\max _{i \in 1, \ldots, n}\left|u_{i}-u_{i}^{(k+1)}\right|_{L B}$ & $\left|u-u^{(k+1)}\right|_{U B}$ & $\max _{i \in 1, \ldots, n}\left|u_{i}-u_{i}^{(k+1)}\right|_{U B}$ \\
\hline \hline$C=3.51$ & 0.5 & 0.0309 & 0.0098 & 0.3187 & 0.1000 \\
$a=1$ & -0.4 & 0.0718 & 0.0226 & 0.2778 & 0.0872 \\
& 0.4 & 0.0878 & 0.0276 & 0.2618 & 0.0821 \\
& -0.08 & 0.0416 & 0.0145 & 0.3035 & 0.0952 \\
\hline$C=3.51$ & 0.5 & 0.1977 & 0.0622 & 0.1519 & 0.0476 \\
$a=3$ & -0.4 & 0.2946 & 0.0926 & 0.0550 & 0.0172 \\
& 0.4 & 0.2467 & 0.0776 & 0.1029 & 0.0322 \\
& -0.08 & 0.2728 & 0.0858 & 0.0767 & 0.0240 \\
\hline \hline
\end{tabular}

Table 5: Errors of new and known methods on Bratu problem for $C=3.51$

iteration, where Homeier's method, $\gamma=0.5$, diverges when it tries to approach both branches of the solution, $\gamma=-0.08$ converges to the solution at the lower branch but not to the one at the upper branch and the rest of schemes, $\gamma= \pm 0.4$, provide reasonable results, showing the expected good stability properties.

\begin{tabular}{|c|c|c|c|}
\hline & $\gamma$ & $\left\|u^{(k)}-u^{(k-1)}\right\|$ & $\left\|F\left(u^{(k)}\right)\right\|$ \\
\hline \hline$C=3.5138$ & 0.5 & 20.42 & $3.44 \mathrm{e}+149$ \\
$a=1$ & -0.4 & 0.2391 & $1.11 \mathrm{e}-04$ \\
& 0.4 & 1.1880 & $3.88 \mathrm{e}-04$ \\
& -0.08 & 0.4631 & $6.65 \mathrm{e}-04$ \\
\hline$C=3.5138$ & 0.5 & 89.51 & $1.01 \mathrm{e}+17$ \\
$a=3$ & -0.4 & 0.2245 & $1.12 \mathrm{e}-04$ \\
& 0.4 & 0.6585 & $5.27 \mathrm{e}-04$ \\
& -0.08 & 4.8310 & $1.39 \mathrm{e}+46$ \\
\hline \hline
\end{tabular}

Table 6: Approximating results of new and known methods on Bratu problem for $C=3.5138$

Results in Table 7 show that, even in almost singular cases, the converging proposed methods yield good enough results, in terms of proximity to the exact solution (the best ones are again $\gamma= \pm 0.4$ ). In this case, lower and upper branches are close and the respective exact errors are similar simultaneously in both branches, for both values of parameter $a$.

\section{Conclusions}

A family of parametric iterative methods of order three, that contains Homeier scheme is constructed. The dynamical behavior of the designed family on quadratic polynomials is very rich. From parameter planes, it has been proved that there are many values of parameter $\gamma$, that is, elements of the family, with no convergence to 


\begin{tabular}{|c|c|c|c|c|c|}
\hline & $\gamma$ & $\left\|u-u^{(k+1)}\right\|_{L B}$ & $\max _{i \in 1, \ldots, n}\left|u_{i}-u_{i}^{(k+1)}\right|_{L B}$ & $\left|u-u^{(k+1)}\right|_{U B}$ & $\max _{i \in 1, \ldots, n}\left|u_{i}-u_{i}^{(k+1)}\right|_{U B}$ \\
\hline \hline$C=3.5138$ & 0.5 & 496.20 & 348.79 & 496.17 & 348.79 \\
$a=1$ & -0.4 & 0.0048 & 0.0014 & 0.0824 & 0.0258 \\
& 0.4 & 0.3221 & 0.1012 & 0.2445 & 0.0768 \\
& -0.08 & 0.4410 & 0.1386 & 0.3634 & 0.1142 \\
\hline$C=3.5138$ & 0.5 & 108.36 & 46.36 & 108.30 & 46.36 \\
$a=3$ & -0.4 & 0.0603 & 0.0190 & 0.0152 & 0.0049 \\
& 0.4 & 0.3509 & 0.1101 & 0.3960 & 0.1243 \\
& -0.08 & 252.59 & 109.81 & 252.56 & 109.79 \\
\hline \hline
\end{tabular}

Table 7: Errors of new and known methods on Bratu problem for $C=3.5138$

the roots. The existence of periodic orbits of period two and four has been showed and its analytical expression has been obtained in terms of parameter $\gamma$. Nevertheless, these black areas of no convergence to the roots are very small compared with the respective ones of classical iterative families as King's or Jarratt's. It is also important to remark that some of the unstable areas found in $P_{1}$ correspond to complex values of the parameter $\gamma$, which are rarely used, and therefore the great majority of the parameter space correspond to iterative methods with a very good numerical behavior. Finally, some of this methods are used to solve the planar 1-D Bratu problem, obtaining great results under singular conditions.

\section{Aknowledgements}

The authors would like to thank the anonymous reviewers for their valuable suggestions and comments that have substantially improved the final version of this manuscript.

\section{References}

[1] S. Amat, S. Busquier, C. Bermúdez, Á.A. Magreñán, On the election of the damped parameter of a two-step relaxed Newton-type method, Nonlinear Dynamics, DOI 10.1007/s11071-015-2179-x

[2] S. Amat, S. Busquier, C. Bermúdez, S. Plaza, On two families of high order Newton type methods, Appl. Math. Letters, 25, 2209-2217, (2012)

[3] S. Amat, S. Busquier, S. Plaza, Review of some iterative root-finding methods from a dynamical point of view, Sci. Ser. A: Math. Sci., 10, 3-35, (2004)

[4] D.K.R. Babajee, A. Cordero, J.R. Torregrosa, Study of iterative methods through the Cayley Quadratic Test, J. of Comput. and Appl. Math., 291, 358-369, (2016)

[5] DKR Babajee, R. Thukral, On a 4-Point Sixteenth-Order King Family of Iterative Methods for Solving Nonlinear Equations, Int. J. Math. Math. Sci. Volume 2012, ID 979245, 13 pages, (2012)

[6] P. Blanchard, Complex Analytic Dynamics on the Riemann Sphere, Bull. of the AMS, 11(1), 85-141, (1984)

[7] P. Blanchard, The Dynamics of Newton's Method, Proc. of Symp. in Appl. Math., 49, 139-154, (1994)

[8] J.P. Boyd, One-point pseudospectral collocation for the one-dimensional Bratu equation, Appl. Math. Comput., 217, 5553-5565, (2011)

[9] G. Bratu, Sur les equation integrals non-lineaires, Bull. Math. Soc. France, 42, 113-142, (1914)

[10] F. Chicharro, A. Cordero, J.R. Torregrosa, Drawing dynamical and parameter planes of iterative families and methods, The Sci. World J., 2013, Article ID 780153, (2013)

[11] C. Chun, M.Y. Lee, A new optimal eighth-order family of iterative methods for the solution of nonlinear equations, Appl. Math. Comput., 223, 506-519, (2013) 
[12] A. Cordero, J. García-Maimó, J.R. Torregrosa, M.P. Vassileva and P. Vindel, Chaos in King's iterative family, Appl. Math. Lett., 26, 842-848, (2013)

[13] A. Cordero, J.R. Torregrosa, Variants of Newton's method using fifth-order quadrature formulas, Appl. Math. Comput., 190, 686-698, (2007)

[14] A. Cordero, J.R. Torregrosa and P. Vindel, Dynamics of a family of Chebyshev-Halley type method, Appl. Math. Comput., 219, 8568-8583, (2013)

[15] P. Fatou, Sur les quations fonctionelles, Bull. Soc. mat. Fr., 47, 161-271, (1919); 48, 33-94; 208-314,(1920)

[16] I.M. Gelfand, Some problems in the theory of quasi-linear equations, Trans. Amer. Math. Soc. Ser., 2, 295-381, (1963)

[17] J.M. Gutiérrez, M.A. Hernández and N. Romero, Dynamics of a new family of iterative processes for quadratic polynomials, J. of Comput. and Appl. Math., 233, 2688-2695, (2010)

[18] H.H.H. Homeier, On Newton-type methods with cubic convergence, J. Comput. Appl. Math., 176, 425-432, $(2005)$

[19] J. Jacobsen, K. Schmitt, The Liouville-Bratu-Gelfand problem for radial operators, J. Differential Equations, 184, 283-298,(2002)

[20] R. Jalilian, Non-polynomial spline method for solving Bratu's problem, Comput. Phys. Comm., 181, 1868$\underline{1872,(2010)}$

[21] G. Julia, Mémoire sur l'iteration des fonctions rationnelles, J. Mat. Pur. Appl., 8, 47-245,(1918)

[22] Á. A. Magreñán, Different anomalies in a Jarratt family of iterative root-finding methods, App. Math. Comput., 233, 29-38, (2014)

[23] A. Mohsen, A simple solution of the Bratu problem, Computers and Mathematics with Applications, 67, 26-33, (2014)

[24] B. Neta, C. Chun, M. Scott, Basins of attraction for optimal eighth order methods to find simple roots of nonlinear equation, App. Math. Comput., 227, 567-592, (2014)

[25] A.M. Ostrowski, Solution of equations and systems of equations, Academic Press, New York, 1960.

[26] M. Petković, B. Neta, L.D. Petković, J. Džunić, Multipoint Methods for Solving Nonlinear Equations, Academic Press, Amsterdam, 2013.

[27] M. Scott, B. Neta, C. Chun, Basin attractors for various methods, Appl. Math. and Comput., 218, 25842599, (2011)

[28] J.R. Sharma, Improved Chebyshev-Halley method with sixth and eighth order of convergence, Appl. Math. Comput., 256, 119-124, (2015)

[29] J.L. Varona, Graphic and numerical comparison between iterative methods, Math. Intelligencer, 24, 37-46, $\underline{(2002)}$

[30] Y.Q. Wan, Q. Guo, N. Pan, Thermo-electro-hydrodynamic model for electrospinning process, Int. J. Nonlinear Sci. Numer. Simul., 5, 5-8, (2004) 